\title{
Analysis of Piezoelectric Solids through Boundary Element Method
}

\section{Qing H. Qin*}

Research School of Engineering, Australian National University, Canberra, Australia

Boundary element method (BEM) is a powerful computational tool for analysing piezoelectric problems. The BEM has been so well developed during the past 40 years that it has been considered as a very popular computational tool. This method consists of formulating the engineering problem in terms of an integral equations relating only boundary values and determining its solutions numerically. Thus, it requires only a surface discretization, rather than a full-domain discretization with "domain-type techniques, such as the finite element method. If interior domain values are needed, however, it can be subsequently calculated from boundary data. Remarkable advances in this area during last decades can be found, for example, in Manolis and Beskos[1] and Qin [2,3]. For piezoelectric problems, BEM, as an important complementary tool for analytical method [4-8], has been the subject of fruitful scientific attention by many a distinguished researcher e.g. Lee and Jiang [9], Denda and Mansukh [10], Sanz, Ariza and Dominguez [11], Lee and Jiang [9] derived the boundary integral equation of piezoelectric media by the method of weighted residuals for plane piezoelectricity. Lu and Mahrenholtz [12] presented a variational boundary integral equation for the same problem. Ding, Wang and Chen [13] developed a boundary integral formulation which is efficient for analysing crack problems in piezoelectric material. Xu and Rajapakse $[14,15]$ presented the formulations for problems of piezoelectric solids with various defects (cavities, inclusions, cracks, etc.). Pan [16] derived a single domain $\mathrm{BE}$ formulation for $2 \mathrm{D}$ static crack problems. Denda and Lua [17] developed a BEM formulation using Stroh's formalism to derive the fundamental solution without numerical results. Qin [18] presented a BEM formulation for cracked piezoelectric materials with half-plane boundary. Later, Qin and Lu [19] extended the model presented in [18] to the case of piezoelectric materials containing both crack and inclusion problems. Davi and Molazo [20] used the known sub domain method to formulate a multi-domain BEM, well suited for crack problems, by modelling crack faces as boundaries of the different sub domains. Groh and Kuma [21] developed a direct collocation boundary element code with a sub domain technique for analysing crack problems and calculating stress intensity factors. Qin an Mai [22] investigate crack-hole interaction of piezoelectric materials using BEM. Zhao et al. [23], presented a boundary integral-differential model for interfacial cracks in 3D piezoelectric solids. Qin [24,25] imbedded BEM into micromechanics model for evaluating effective properties of piezoelectric solids.

There are three major approaches in the literature for establishing boundary integral equations of piezoelectric materials, i.e., the variational approach [12], weighted residual approach [9], and Betti's reciprocity theorem [16]. A brief description on the above three approaches which have been widely used in BE analysis is given here. (1) The generalized variational method is based on a modified functional with six kinds of independent variable, i.e. displacements and electric potential in the domain, and displacements, tractions, electric potential and surface charge on the boundary. Since all the boundary conditions considered have been introduced into the framework of the modified variational expression, no additional constraints have to be satisfied when assuming the variables on the boundary; (2) The weighted residual approach is a general method of establishing numerical algorithms like finite element and boundary element method. Different weighted function may lead to different numerical method. If the fundamental solution is employed as a weighted function in a potential energy functional, a boundary element formulation can be established. As indicated in [9], the weighted residual statement for the boundary value problem of piezoelectric materials may be expressed as

$$
\begin{aligned}
\int_{\Omega}\left(\sigma_{i j, j}^{*} u_{i}+D_{i, i}^{*} \phi+b_{i} u_{i}^{*}+b_{e} \phi^{*}\right) d \Omega & =\int_{\Gamma_{t}}\left(t_{i}^{*} u_{i}-\bar{t}_{i} u_{i}^{*}\right) d \Gamma+\int_{\Gamma_{u}}\left(\bar{u}_{i} t_{i}^{*}-u_{i}^{*} t_{i}\right) d \Gamma \\
& +\int_{\Gamma_{\phi}}\left(q_{s}^{*} \bar{\phi}-\phi^{*} q_{s}\right) d \Gamma-\int_{\Gamma_{D}}\left(\bar{q}_{s} \phi^{*}-q_{s}^{*} \phi\right) d \Gamma
\end{aligned}
$$

where $\sigma_{i j}$ is stress component, $u_{i}$ mechanical displacement, $D_{i}$ electric displacement, $\phi$ electric potential, $t_{i}$, and $q_{s}$ are the surface traction and surface charge, $b_{i}$ and $b_{e}$ are the body force per unit volume and electric charge density, a bar over a variable means that variable is a prescribed, a superscript " »" means the associated variable is the corresponding fundamental solution, $\Omega$ is the solution domain bounded by $\Gamma$. A comma followed by an index means differentiation, $\Gamma_{u}, \Gamma_{\phi}, \Gamma_{t}$, and $\Gamma_{D}$ are the boundaries on which the prescribe $u, \phi, t$, and $\mathrm{D}$ are imposed; and (3) In addition to the variational approach and weighted residual methods discussed above, the reciprocity theorem can also be used to establish the boundary integral equation. Please refer to [17]for the details on applications of this method to piezoelectric materials.

BEM can also be applied to piezoelectric composites in conjunction with homogenization or micromechanics approach for determining their effective material properties [24,25]. Micromechanics theories usually employ the well-known stress and strain concentration factors obtained through an analytical solution of a single crack, or void, or inclusion embedded in an infinite medium. However, for a problem with complexity in the aspects of geometry and mechanical deformation, a combination of these theories and numerical methods such as BEM presents a powerful computational tool for estimating effective material properties. The Micromechanics-BE approach develop in [24] is based on using a representative volume model for materials with defects and introducing it into a $\mathrm{BE}$ formulation to provide an effective means for estimating overall material constants of the defected materials. The micromechanics method produces formulas for overall material constants as functions of a concentration matrix. The concentration matrix is in turn related to the boundary displacement. The boundary element simulation presents numerical solutions of boundary displacement and electric potential for crack, hole, and/or inclusion problems.

*Corresponding author: Qing H. Qin, Research School of Engineering, Australian National University, Canberra, ACT 2601, Australia, E-mail: Qinghua.qin@anu.edu.au

Received November 09, 2012; Accepted November 12, 2012; Published November 15, 2012

Citation: Qin QH (2012) Analysis of Piezoelectric Solids through Boundary Element Method. J Appl Mech Eng 2:e113. doi:10.4172/2168-9873.1000e113

Copyright: () 2012 Qin QH. This is an open-access article distributed under the terms of the Creative Commons Attribution License, which permits unrestricted use, distribution, and reproduction in any medium, provided the original author and source are credited. 
Citation: Qin QH (2012) Analysis of Piezoelectric Solids through Boundary Element Method. J Appl Mech Eng 2:e113. doi:10.4172/21689873.1000 e113

\section{References}

1. Manolis GD, Beskos DE (1983) Dynamic-response of lined tunnels by an isoparametric boundary element method. Comput Method Appl M 36: 291-307.

2. Qin $Q$, Huang $Y(1990)$ BEM of postbuckling analysis of thin plates. App Math Model 14: 544-548.

3. Qin Q (1993) Nonlinear analysis of Reissner plates on an elastic foundation by the BEM. Int J Solid Struct 30: 3101-3111.

4. Qin QH, Mai YW, Yu SW(1999) Some problems in plane thermopiezoelectric materials with holes. Int J Solid Struct 36: 427-439.

5. Yu SW, Qin QH (1996) Damage analysis of thermopiezoelectric properties: Part II. Effective crack model. Theor App Frac Mech 25: 279-288.

6. Qin QH, Mai YW, Yu SW(1998) Effective moduli for thermopiezoelectric materials with microcracks. Int J Fract 91: 359-371.

7. Qin QH, Zhang X (2000) Crack deflection at an interface between dissimilar piezoelectric materials. Int J Fract 102: 355-370.

8. Qin QH, Mai YW (1998) Multiple cracks in thermoelectroelastic bimaterials Theor App Fract Mech 29: 141-150.

9. Lee JS, Jiang LZ (1994) A boundary integral formulation and 2d fundamental solution for piezoelastic media. Mechanics Research Communications 21: 4754.

10. Denda M, Mansukh M (2005) Upper and lower bounds analysis of electric induction intensity factors for multiple piezoelectric cracks by the BEM. Eng Anal Bound Elem 29: 533-550.

11. Sanz JA, Ariza MP, Dominguez J (2005) Three-dimensional BEM for piezoelectric fracture analysis. Eng Anal Bound Elem 29: 586-596.

12. Lu P, Mahrenholtz O (1994) A variational boundary-element formulation for piezoelectricity. Mech Res Commun 21: 605-611.
13. Ding HJ, Wang GQ, Chen WQ (1998) A boundary integral formulation and 2D fundamental solutions for piezoelectric media. Comput Method App Mech Eng 158: $65-80$.

14. Xu XL, Rajapakse RKND (1998) Boundary element analysis of piezoelectric solids with defects. Composites Part B-Eng 29: 655-669.

15. Rajapakse RKND, Xu XL (2001) Boundary element modeling of cracks in piezoelectric solids. Eng Anal Bound Elem 25: 771-781.

16. Pan E (1999) A BEM analysis of fracture mechanics in $2 D$ anisotropic piezoelectric solids. Eng Anal Bound Elem 23: 67-76.

17. Denda M, Lua J (1999) Development of the boundary element method for 2D piezoelectricity. Compos Part B Eng 30: 699-707.

18. Qin QH (1999) Thermoelectroelastic analysis of cracks in piezoelectric halfplane by BEM. Comput Mech 23: 353-360.

19. Qin QH, Lu M (2000) BEM for crack-inclusion problems of plane thermopiezoelectric solids. Int J Num Method Eng 48: 1071-1088.

20. Davi G, Milazzo A (2001) Multidomain boundary integral formulation for piezoelectric materials fracture mechanics. Int J Solid Struct 38: 7065-7078.

21. Groh U, Kuna M (2005) Efficient boundary element analysis of cracks in 2D piezoelectric structures. Int J Solid Struct 42: 2399-2416.

22. Qin QH, Mai YW (2002) BEM for crack-hole problems in thermopiezoelectric materials. Eng Frac Mech 69: 577-588.

23. Zhao MH, Fang PZ, Shen YP (2004) Boundary integral-differential equations and boundary element method for interfacial cracks in three-dimensional piezoelectric media. Eng Anal Bound Elem 28: 753-762.

24. Qin QH (2004) Micromechanics-BE solution for properties of piezoelectric materials with defects. Eng Anal Bound Elem 28: 809-814.

25. Qin QH (2004) Material properties of piezoelectric composites by BEM and homogenization method. Comp Struct 66: 295-299. 